\title{
SCREENING PROTOCOL FOR SIGNS OF AUTISTIC SPECTRUM DISORDER FOR BABIES
}

\author{
Carolina Alcântara, \& Laura Melo \\ SARAH Network of Neurorehabilitation (Brazil)
}

\begin{abstract}
The objective of the present work is to present a proposal of a Screening Protocol for Signs of Autism Spectrum Disorder for babies. The motivation for the development of this work lies in the clinical practice of rehabilitation and care for babies at the Sarah Hospital of Rehabilitation of São Luís. The change in the profile of care to this population implied the need to systematize the skills of the team for specialized assessment of development. The method was outlined based on a review of specific literature in the field, in particular Gadia's studies, data from the Brazilian Society of Pediatrics, in accordance with international evaluation parameters. Observation was used as a methodological procedure. As a result, an evaluation systematics has been established that points out objective criteria for early insertion in a program of stimulation and / or referral to specialized services, enabling interventions that promote changes in patterns of behavior and development. The tool includes a qualitative assessment of social development and language milestones in infants under 18 months of age; application of Screening for early screening between 18 to 24 months of age; performing complementary screening tests; and standardized assessment of development through specific scale application.
\end{abstract}

Keywords: Autism spectrum disorder, babies, child development.

\section{Introduction}

The aim of this paper is to present a proposal for Medical Screening Protocol for Signs of Autistic Spectrum Disorder in Babies. The development of this work was motivated by the clinical practice of rehabilitation and care for babies at Sarah Rehabilitation Hospital, in São Luis. There has been a significant increase in the number of babies treated at the institution since 2015, with the beginning of the Program for Admission of Babies via internet. The change in procedures of taking care of this population implied the need to systematize the Hospital team's repertoire for specialized development assessment, aiming the early identification of problem behaviors and signs of delay in neurodevelopment, consequently early initiation of a stimulation program and / or specialized referral.

According to DSM V, Diagnostic and Statistical Manual of Mental Disorders, Autistic Spectrum Disorder (ASD) may be characterized by persistent deficits in social communication and interaction in multiple contexts, including deficit in social reciprocity and nonverbal behaviors used for social interaction and in skills for developing, maintaining and understanding relationships (American Psychiatric Association, 2014).

There is also reference to restricted and repetitive patterns of behavior, interests or activities. Among the established diagnostic criteria, there are specifiers of the beginning of symptoms that must be present early in the development period, which shows that problem behaviors are usually recognized at the age of 12 months, although they can be observed before this age if developmental delay is severe or failures in critical behavior are more evident.

Contributions of Gadia and other authors on the assessment of ASD signs at an early age are listed here as reference and guideline for this work, motivated by the understanding this author has pointed to the need for developmental pediatricians to be involved in the process of identifying behavioral signals which are relevant to the longitudinal diagnostic process of ASD. Gadia presents the importance of pediatricians, in interface with areas of child development, recognizing developmental skills in the evaluation process.

Initially, this work lies in an interdisciplinary understanding of assessment and analysis of child development, as only it can provide adequate care for children with neurodevelopmental disorders. It is understood that as important as the availability and accuracy of the practice of professionals, who already work directly in the area of child development, is the early identification by other professionals who work in the initial sphere of monitoring, medical screening services and initial evaluation of pediatrics. 
This interface of action between areas of knowledge can optimize the progress of clinical follow-up of infants who have neurodevelopmental and / or neuropsychiatric disorders. In addition to the paralyzing diagnostic search, there is the diagnostic search that alters paths and focuses on what we call resilience. It alters vulnerability and risk, transforming the possibility of quality of life of babies, children and their families.

The Sarah Network of Neurorehabilitation is reference in the treatment of neurological problems in children and adults in Brazil. It consists of nine units and serves patients from all over the country. In childhood, most of the patients are diagnosed with cerebral palsy or traumatic brain injury. Children and adolescents are treated through many modalities by an interdisciplinary health team. The São Luís, Maranhão unit is located in the neighborhood of Monte Castelo and has the Neurological Rehabilitation in Spinal Cord Injury, Neurological Rehabilitation and Orthopedics Programs, where adults and children are admitted.

\section{Methodology}

The method was based on a review of specific literature in the area of knowledge that points to the interface between pediatrics and development, especially Gadia studies, by authors addressing the importance of early assessment and stimulation in ASD, current data from the Diagnostic Manual and Mental Disorders Statistics (DSM V) and information recommended by the Brazilian Society of Pediatrics, in accordance with international assessment parameters. In addition, observation was used as a methodological procedure.

\section{Results}

As a result, a screening protocol for signs of autistic spectrum disorder has been developed to be used in the clinical practice of the hospital rehabilitation team. Therefore, there is a simplified evaluation system that aims at objective criteria for early insertion in stimulation and / or referral to specialized services, enabling interventions that promote changes in behavioral and development patterns and increase in the quality of babies' and their families' lives. The tool includes qualitative assessment of social and language development in infants younger than 18 months; Screening application for early diagnosis in children between 18 and 24 months of age and complementary exams in addition to standardized assessment of development through specific scale application.

It is relevant to note that the protocol obtained has been used in clinical practice paired with the other qualitative data that are recorded by the hospital interdisciplinary team during care, as already practiced at the Child Rehabilitation Center. The qualitative aspects narrated by family members are part of all clinical procedures performed and are saved in an electronic medical record, a registration and monitoring tool used by the team.

Specific information about the developed Protocol is presented below and includes qualitative assessments of behavior and development (based on family history and direct observation of assisted infants), application of the Modified Checklist for Autism in Toddlers (M-CHAT) scale for infants between 18 and 24 months of age, additional tests requested by the pediatrician, and the possible use, according to the hospital team's analysis, of the Bayley Scales Test of Infant and Toddler Development.

\subsection{Screening protocol for autistic spectrum disorder signals in infants}

Assessment of infant development is one of the key roles of the hospital interdisciplinary rehabilitation team in the care process for infants at risk. This evaluation aims to point out global development acquisitions and correlates to developmental markers that allow the identification of a strengthened repertoire in the process of constitution or the detection of flaws that suggest development delay.

Commonly assessed axes involve global cognition, language, motor development, and behavioral framework. Based on the analysis of literature regarding developmental signs possibly related to ASD, we highlight as evaluation system for babies up to 18 months of age, evaluations that are significant for the hospital team analysis. This evaluation makes up the first screening stage.

\subsection{Qualitative assessment of development, social interaction and language in infants up to 18 months}

a) eye contact analysis: observation of the ability to initiate and maintain visual response to the contact of the interlocutor and quality of eye contact. Observe if there is avoidance or vague look, peculiar characteristics in the way the child looks at objects;

b) analysis of social interaction schemes: observation of the skills to establish social exchanges, participation in simple interactions, transmission and response to social smile. Observation of interest in 
social stimuli, including behavioral fixation of the human face, response modification upon presentation of specific social stimuli (e.g. caregivers);

c) analysis of the language development stage: discrimination of signs that suggest delay; observation of sounds as well as features of language functionality. Discrimination of atypical language processes, persistence of expressive jargon at an unexpected stage. Evaluation of verbal and nonverbal communication repertoire;

d) analysis of auditory responses: discrimination of signs of changes in responsiveness to auditory stimuli, sound response, source location. Evaluation of changes in the responsiveness of sounds of physical and social environment;

e) assessment of social imitation behaviors: evaluation of immediate imitation sound schemes or motor schemes as expected in global development. Assess whether imitation responses occur spontaneously or require support from others;

f) assessment of shared attention schemes: assessment of behaviors that indicate the child's ability to follow social stimulus toward other environmental stimuli, as well as the ability to sustain interaction with stimuli presented by the interlocutor;

g) evaluation of symbolic thinking schemes: evaluation of behaviors during play that indicate ability for symbolic representation, either from imitation behaviors or spontaneously in interaction with objects arranged in the environment or imaginary objects;

h) motor development analysis: assessment of the overall motor repertoire; identification of signs of delay or atypical, stereotyped and poorly functioning motor behavior.

The objectives of systematizing these evaluation axes are aimed at favoring the observation of behaviors and critical developmental skills by professionals of the hospital interdisciplinary team. However, it is understood that the identification of the previously mentioned skills is not conclusive of a priori diagnoses, making it fundamental to contextualize and analyze the global development which allows an interpretation of the observed data.

\subsection{Initial evaluation}

\section{a) Application of the M-CHAT Scale for babies aged 18 to 24 months}

In the present study, the data expressed in the methodology, were sought to support the use of standardized tools for assessing signs of ASD in infants. Within this scenario, considering the assumed relevance to clinical practice, the use of the M-CHAT Scale aimed at children aged between 18 and 24 months, version translated into Portuguese by Losapio and Pondé (2008).

M-CHAT is a screener to assess ASD traits in young children. It is a simple tool that can be used by pediatrics and other health professionals, and can point out signs of behavior or development that point to critical flaws that indicate the need for specialized diagnostic evaluation (Losapio \& Pondé, 2008).

The instrument should be applied to parents or caregivers and has high sensitivity and specificity. It is made up of 23 yes / no questions, is easily available in electronic version and points to the final impression of whether there is a risk for ASD or not. It is a screening tool, not a diagnostic tool, which facilitates the identification of situations to be better investigated in services in ASD (Brazilian Society of Pediatrics, 2017).

The application of the M-CHAT scale is directed to babies between 18 and 24 months of age, since literature has indicated that its application at an earlier age is more strongly associated with false positives. Thus, we opted only for qualitative analysis of behavior / development stages up to 18 months (as presented in the previous item) and, from this age on, complementary application of the M-CHAT scale in all infants treated.

The objective of choosing this instrument was to achieve a quantitative data that complements the assessment performed by the hospital team and supports, together with other evaluations and complementary exams, the definition of follow-up conducts either in stimulation in the program and / or referral to specialized services.

b) Evaluation of complementary exams and discussion with pediatrics

Clinical assessment and stimulation of infants at risk also points to the need for additional tests to support important differential diagnoses in developmental clinics, enabling further elucidation between primary sensorial changes, global development delay, language disorders, TEA, among others. below:

Significant complementary exams for the screening stage and initial investigation are listed Audiometry (BERA);

- Behavioral auditory assessment: this is a complementary research tool with qualitative basis, whose data are measured in situations of interaction with the child and discussed with the hospital speech therapy team;

- Neuroimaging: for those who have risk factors for brain injury, in pre-, peri- and postnatal history; 
- Molecular biology: Requested for those without etiology for ASD, consists of mutation (deletion / duplication) research in regions $15 \mathrm{q} 11-13$ and $16 \mathrm{p} 11$ and $22 \mathrm{q} 13$ by the Multiplex Ligation-Dependent Probe Amplification (MLPA) technique and relative quantification of regions 15q11-13 and 16p11 and 22q13. Cytogenetically visible chromosomal changes, such as rearrangements, deletions, and duplications, have been identified in 3 to $5 \%$ of ASD patients, while microdeletions are found in approximately $10 \%$ of cases (Vorstman et al., 2006; Morrow et al., 2008). Among the chromosomal changes most commonly found in ASD patients are deletions and duplications in 15q11-13 (Sharp et al., 2005; Pagnamenta et al., 2009), 16p11 deletions (Ballif et al., 2007; Kumar et al. 2008; Marshall et al., 2008) and 22q13 deletions (Moessner et al., 2007; Sykes et al., 2009). The MLPA technique allows the search for mutations, specifically deletions and duplications in these three described regions.

\section{c) Development Assessment}

The assessment of global development consists of the analysis of the acquisition of cognitive, motor and language axes and is performed by the interdisciplinary rehabilitation team from qualitative analysis frameworks and ecological development perspective, and may also have standardized tools for analysis. It also allows deepening on the significant behavioral data measured in screening instruments or resulting from family history.

In the studied clinical practice, the hospital team opted to use the Bayley Scale Test as a standardized tool. The Bayley Scale Test is a tool for screening cognitive, motor and language delay in infants from 1 to 42 months of age. It is an instrument that is easy and quick to administer and is therefore usable in hospital and outpatient settings. The application enables testing of different development domains and inference as to the indication of a more extensive and comprehensive assessment of development. In the evaluation context, the baby is encouraged to participate in playful activities and the evaluation data are compared with cutoffs according to age group.

\section{d) Orthopedic evaluation}

In addition to the abovementioned assessments, all infants treated are evaluated for the indication of participation and follow-up in a protocol of gait digression, with the orthopedics team, using triceps shortening criteria.

\section{e) Medical Referral Criteria}

After behavioral analysis, development and complementary tests that favor differential diagnosis, in case of positive impression of signs of ASD, all evaluated babies are referred to a specialized diagnostic service for diagnostic elucidation (neuropediatrician or infant psychiatrist), based on data given by the global team assessment. In addition, infants with brain injury up to 18 months of age are referred to the developmental stimulation group with the interdisciplinary team in the Child Rehabilitation program.

Confirmed diagnostic data are later saved in a specific electronic medical record icon for proper notification and database recording for future studies.

The proposal of the signal assessment protocol for ASD previously described has been implemented within the context of Child Rehabilitation at the present service. At this stage, in which the initial protocol proposal was concluded and its application started in the clinical context, there is a positive analysis by the interdisciplinary team about its effectiveness, especially regarding a more objective and standardized documentation of the development and clinical aspects of these babies, enabling even greater safety on the part of the team in the conduct definition process, either by indicating with more objective goals for the stimulation program (discrimination of target behaviors and developmental skills to be achieved) or by defining regarding the need for referral to specialized service, when necessary.

In future studies, we may also seek to identify results about the correlation between data obtained with the protocol and confirmed diagnostic data for Autistic Spectrum Disorder or other neurodevelopmental changes. It is well known that early intervention programs point to more significant gains for children with these conditions (Lampreia, 2007; Losapio \& Pondé, 2008; Brazilian Society of Pediatrics, 2017).

\section{Final considerations}

This work presents contributions aimed at the qualification of the evaluation in the health service that sometimes constitutes a trigger for a specialized diagnostic evaluation process. It is well known that misinformation and little repertoire for health professionals' assessment of development is one of the main barriers in the early diagnosis process for neurobehavioral disorders, including Autistic Spectrum Disorder.

Although there is documentation of specific instruments with validation for the Brazilian population that are focused on the development of the baby, it is important to note that there are still few specific instruments aimed at assessing signs of development correlated with ASD. The available scales 
for evaluating the development of infants are extremely useful in clinical practice, but usually allow inferences more associated with global development and are not used in most services as isolated predictors of specific behavioral conditions.

The evaluation of signs of Autistic Spectrum Disorder in infants is discussed in specialized literature, so it is known that evaluation at an early stage of development may imply the identification of false positives or interventions in infants who will be evaluated in the future as a group out of the spectrum. In babies with risk factors, such as prematurity, this data is even more relevant. Nevertheless, it is understood that early assessment and intervention can support the structuring of alternative paths for development and benefit qualitatively the repertoire and acquisitions of large groups of infants and children.

\section{References}

American Psychiatric Association. (2014). Manual diagnóstico e estatístico de transtornos mentais: DSM 5 ( $5^{\mathrm{a}}$ ed.). Porto Alegre: Artmed.

Ballif, B. C., Hornor, S. A., Jenkins, E., Madan-Khetarpal, S., Surti, U., Jackson, K. E., Asamoah, A., Brock, P. L., Gowans, G. C., Conway, R. L., Graham Júnior, J. M., Medne, L., Zackai, E. H., Shaikh, T. H., Geoghegan, J., Selzer, R. R., Eis, P. S., Bejjani, B. A., Shaffer, L. G. (2007). Discovery of a previously unrecognized microdeletion syndrome of 16p11.2-p12.2. Nature Genetics, 39, 1071-1073.

Gadia, C., Tuchman, R., \& Rotta, N. (2004). Autismo e doenças invasivas do desenvolvimento. Jornal de Pediatria, 80, 83-94.

Kumar, R. A., KaraMohamed, S., Sudi, J., Conrad, D. F., Brune, C., Badner, J. A., Gilliam, T. C., Nowak, N. J., Cook Junior, E. H., Dobyns, W. B., \& Christian, S. L. (2008). Recurrent $16 p 11.2$ microdeletions in autism. Human Molecular Genetics, 17, 628-638.

Lampreia, C. (2007). A perspectiva desenvolvimentista para a intervenção precoce no autismo. Estudos de Psicologia, 24, 105-114.

Losapio, M., \& Pondé, M. (2008). Tradução para o português da escala M-CHAT para rastreamento precoce de autismo. Revista de Psiquiatria do Rio Grande do Sul, 30, 221-229.

Morrow, E., Yoo, S. Y., Flavell, S. W., Kim, T. K., Lin, Y., Hill, R. S., Mukaddes, N. M., Balkhy, S., Gascon, G., Hashmi, A., Al-Saad, S., Ware, J., Joseph, R. M., Greenblatt, R., Gleason, D., Ertelt, J. A., Apse, K. A., Bodell, A., Partlow, J. N., Barry, B., Yao, H., Markianos, K., Ferland, R. J., Greenberg, M. E., \& Walsh, C. A. (2008). Identifying autism loci and genes by tracing recent shared ancestry. Science, 321, 218-223.

Moessner, R., Marshall, C. R., Sutcliffe, J. S., Skaug, J., Pinto, D., Vincent, J., Zwaigenbaum, L., Fernandez, B., Roberts, W., Szatmari, P., \& Scherer, S. W. (2007). Contribution of SHANK3 mutations to autism sprectrum disorder. American Journal of Human Genetics, 81, 1289-1297.

Pagnamenta, A. T., Wing, K., Sadighi Akha, E., Knight, S. J., Bölte, S., Schmötzer, G., Duketis, E., Poustka, F., Klauck, S. M., Poustka, A., Ragoussis, J., Bailey, A. J., Monaco, A. P., \& International Molecular Genetic Study of Autism Consortium. (2008). A 15q13.3 microdeletion segregating with autism. European Journal of Human Genetics, 17, 687-692.

Sharp, A. J., Locke, D. P., McGrath, S. D., Cheng, Z., Bailey, J. A., Vallente, R. U., Pertz, L. M., Clark, R. A., Schwartz, S., Segraves, R., Oseroff, V. V., Albertson, D. G., Pinkel, D., Eichler, E. E. (2005). Segmental duplications and copy-number variations in the human genome. American Journal of Human Genetics, 77, 78-88.

Sociedade Brasileira de Pediatria. (2017). Triagem Precoce para Autismo/Transtorno do Espectro Autista (Número 1). Rio de Janeiro: SBP.

Sykes, N. H., Toma, C., Wilson, N., Volpi, E. V., Sousa, I., Pagnamenta, A. T., Tancredi, R., Battaglia, A., Maestrini, E., Bailey, A. J., Monaco, A. P., \& International Molecular Genetic Study of Autism Consortium. (2009). Copy number variation and association analysis of SHANK3 as a candidate gene for autism in the IMGSAC collection. European Journal of Human Genetics, 17, 1347-1353.

Vorstman, J., Staal, W. G., van Daale, E., van Engeland, H., Hochstenbach, P. F., \& Franke, L. (2006). Identification of novel autism candidate regions through analysis of reported cytogenetic abnormalities associated with autism. Molecular psychiatry; 11, 18-28. 